\title{
Non-destructive evaluation of sandwich plates by an ultrasonic IR thermographic method
}

\author{
by W. Swiderski*, P. Hlosta* and M. Chuda-Kowalska**
}

* Military Institute of Armament Technology, 7 Wyszynskiego Str., 05-220 Zielonka, Poland, waldemar.swiderski@wp.pl

**Poznan University of Technology, 5 Sq. Marii Sklodowskiej-Curie, 60-965 Poznan, Poland, monika.chudakowalska@put.poznan.pl

\begin{abstract}
The paper presents the possibility of using an ultrasonic IR thermographic method to detect internal defects in composite types of sandwich panels with metal sheets. Experimental results indicate that this method may in many cases be effective in detecting defects that weaken the structure of sandwich panels. The results also show the limitations of using this method to sandwich panels and the materials used in them.
\end{abstract}

\section{Introduction}

Light-weight sandwich panels, made of two external thin and stiff faces, separated by a relatively thick and light core are considered in the paper. These kind of structures are frequently used in civil engineering, especially for wall claddings. That is caused mainly by many of their attractive features like high load-bearing capacity coupled with small weight (because the separation of the skins by the core increases the moment of inertia of the panel with little increase in weight), good thermal insulation, cost of production and easy assembling.

The most common core materials include different kind of cellular solids like metallic, glass, ceramic and polymer foams. For engineering purposes, polymer foams represent an attractive set of features such as high energy absorption capacity, acoustic and thermal insulating properties which make these materials very popular in aircraft, automobile, buildings or packing industry. If the fire protection is needed then mineral wool core is frequently used.

In this work, the main attention was focused on locating and identifying defects in sandwich plates with thick and soft cores. These structures are characterized by completely different mechanical behaviors and failure modes compared to homogeneous structures. Delamination is one of the most common and problematic types of damage occurring both during the manufacturing process as well as during in-service life. It is very difficult to observe this phenomenon in real structures in operation because the damage is not visible on the surfaces (it appears inside structures). However, a lot of laboratory tests were carried out and the effect of delamination appeared in many cases. This phenomenon can have various types depending on action force (bending, shear, pressure) and types of materials of particular layers, as presented in Fig. 1. Debonding and delamination cases presented in this figure are typical for the operation conditions of foam-core sandwich panels in civil engineering.

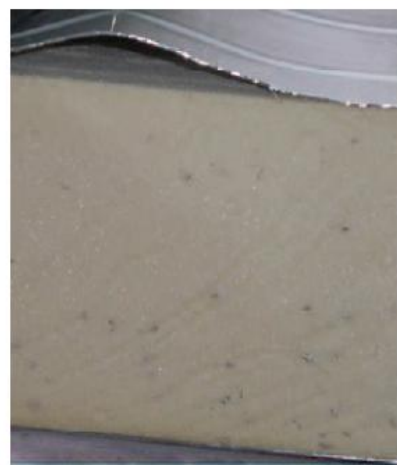

a)

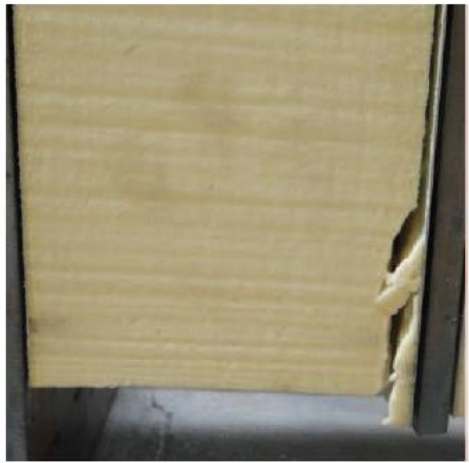

b)

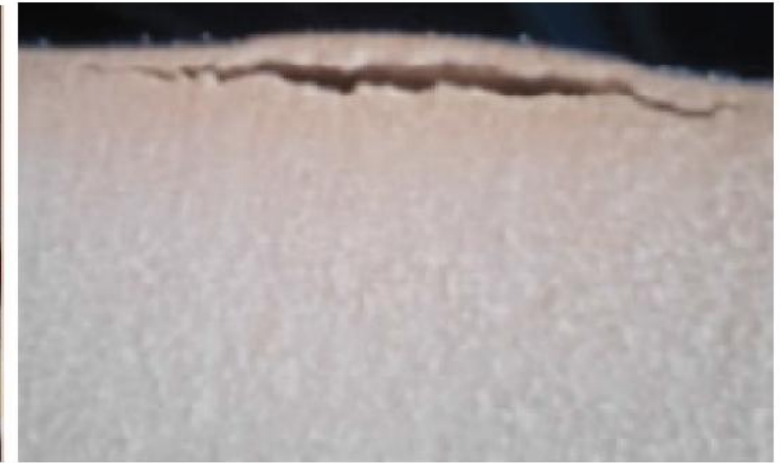

c)

Fig. 1. Various types of delamination caused by: a) bending force, b) shear force, c) pressure

The most common defects include cracks and blisters too. Cracks are associated with a core material failure and are caused by the shear force. Two typical laboratory tests i.e. four point bending test (currently the most common) and direct double-lap shear test are used to obtain this failure. 


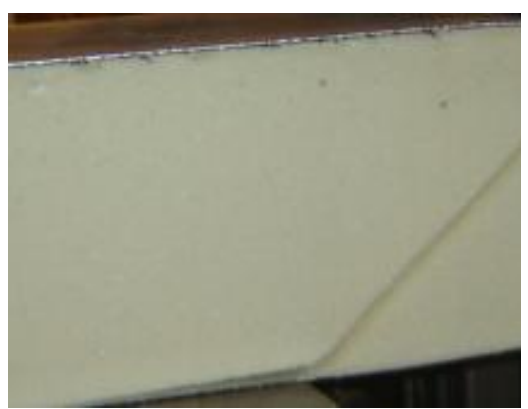

a)

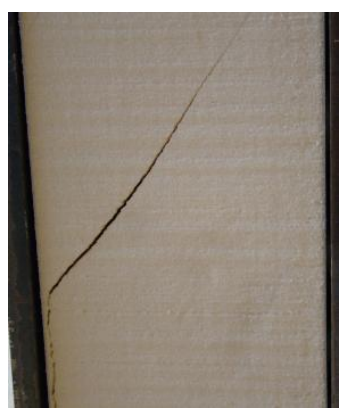

b)

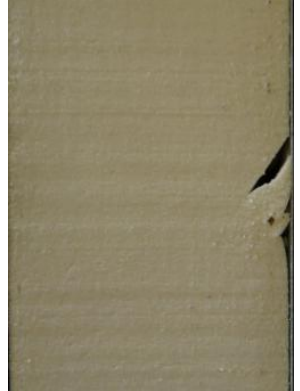

c)

Fig. 2. Various types of cracks caused by: a) bending test b) and c) double lap shear test

It happens that blisters appear in sandwich panels with a foam core and metal facings used in civil engineering. Very often, it is rather a temporary form (disappear when the conditions change for the better) and damage of the plate are rather invisible. Unfortunately, the failure effects can occur in the interface area (Fig. 3) and can be dangerous for further in-service life - local failure is very often the weakest point of the plate and can initiate the damage of the whole structure. The blisters produced artificially are shown in Fig. 3a In this case samples with aluminum facing on the one side and metal facing on the other were tested. During laboratory test pressure inside the sample was introduced. Damages caused by blisters can take different forms and location as presented in Fig. 3b and Fig. 3c.

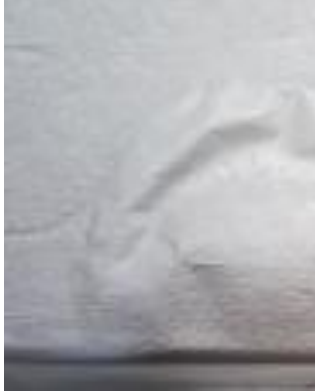

a)

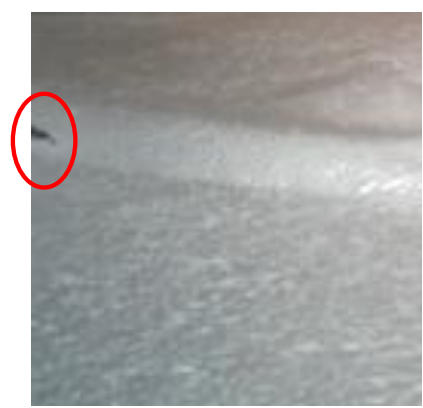

b)

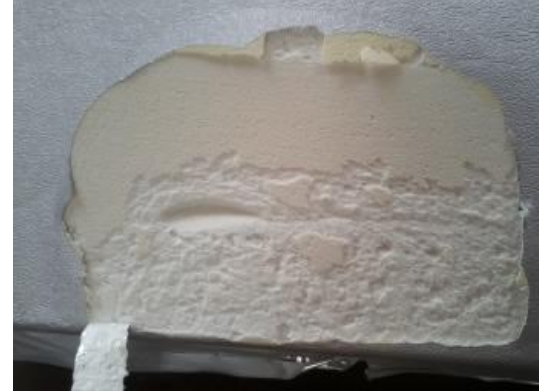

c)

Fig. 3. Various types of blisters : a) blister b) blister with failure of face sheet c) the visible core after removing the blister.

The results of testing sandwich panels covered with glass - epoxy composite sheets using an active thermographic method with stimulation by heat halogen lamps - were presented in the paper [1]. However, this method is not sufficiently effective in the case of sandwich panels with metal sheets. Ultrasonic thermography may be an alternative method for testing these plates.

\section{Mathematical problem}

An ultrasonic IR thermography scheme is presented in Fig. 4.

Theoretical modelling includes two stages:

- Solving a 3D problem concerning the propagation of ultrasonic waves and calculating the heat power generated in defects.

- Solving a 3D problem concerning the propagation of heat from heat sources (defects) which have the characteristics determined during the first stage.

Both of the aforementioned stages are briefly described below. The theoretical approaches described in [2-4] are used in the elaboration of the algorithms below.

An applied thermo-mechanical model implements parallel numerical solutions for the propagation of both thermal energy and mechanical (sonic/ultrasonic) waves. There are both normal and tangent mechanical stresses affecting each facet of an elementary (parallelepiped-like) volume of the tested material (Fig. 5). In turn, these tangent stresses involve two components along two coordinates. Hence, in total, there are three stress components which appear on each parallelepiped surface. For example, on the surface perpendicular to $\boldsymbol{X}$, there is one normal component 
$\sigma_{x}$ and two tangent components $\tau_{x y}$ and $\tau_{x z}$ (the first subscript shows the coordinate axis which is parallel to the normal vector of the parallelepiped surface, and the second subscript indicates the coordinate axis which is parallel to the tangent stress component).

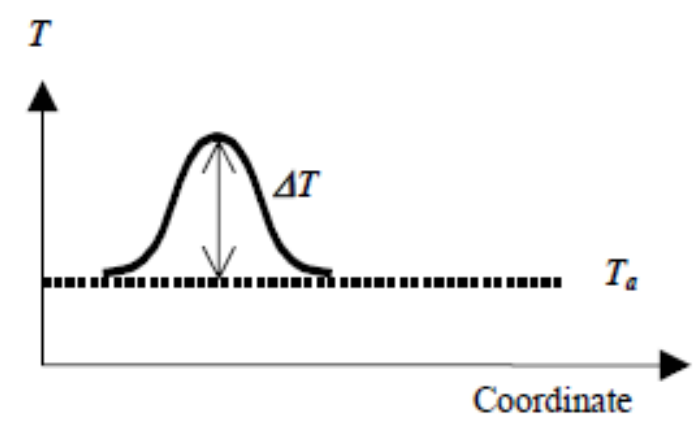

Ultrasonic transducer

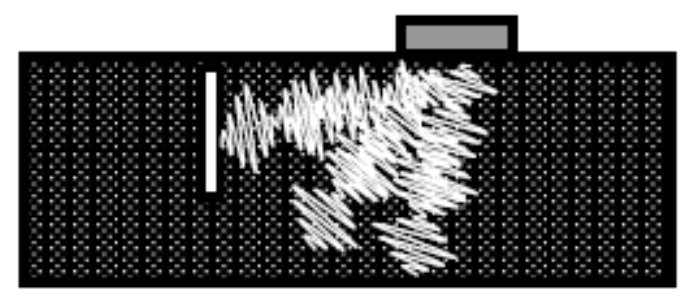

Fig. 4. Ultrasonic IR thermography principle.

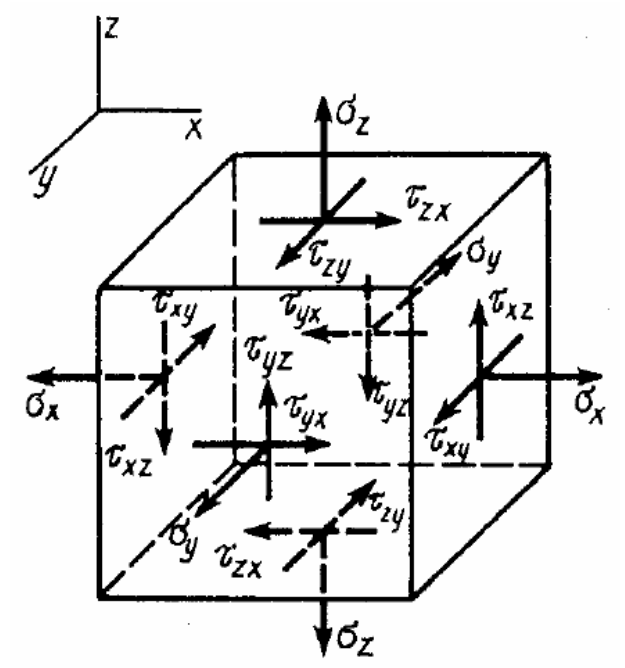

Fig. 5. Stresses acting on a test sample of elementary volume.

In each elementary parallelepiped-like volume of a solid body, there are mechanical stresses acting on each parallelepiped plane i.e., one normal and two tangent stresses (Fig. 5). For example, on the plane perpendicular to the $x$ coordinate, there are $\sigma_{x}, \tau_{x y}$, and $\tau_{x z}$ stresses. The balance of forces along $x$ is given by:

$$
\left(\sigma_{x+0}-\sigma_{x-0}\right) \Delta y \Delta z+\left(\tau_{y+0, x}-\tau_{y-0, z}\right) \Delta x \Delta z+\left(\tau_{z+0, x}-\tau_{z-0, x}\right) \Delta x \Delta y+X \Delta x \Delta y \Delta z=0
$$

where $\Delta x, \Delta y, \Delta z$ are the parallelepiped dimensions, and $X$ is the projection of volumic forces (if they are present) on the $x$ axis. The subscripts $x+0$ and $x-0$ correspond to the maximum and minimum $x$ coordinates.

In a differential form, equation (1) can be reduced to: 


$$
\frac{\partial \sigma_{x}}{\partial x}+\frac{\partial \sigma_{y z}}{\partial y}+\frac{\partial \sigma_{z x}}{\partial z}+X=0
$$

By skipping the mathematics, we arrive at a set of basic equation that has been solved numerically by using ThermoSon software (Tomsk Polytechnic University):

$$
(\lambda+2 G) \frac{\partial^{2} U}{\partial x^{2}}+G\left(\frac{\partial^{2} U}{\partial y^{2}}+\frac{\partial^{2} U}{\partial z^{2}}\right)+(\lambda+G) \frac{\partial^{2} V}{\partial x \partial y}+(\lambda+G) \frac{\partial^{2} W}{\partial x \partial z}=0
$$

where $\lambda$ is the Lamè constant, $G$ is the shear elasticity modulus, and $U, V, W$ are the displacement projections on the $x, y, z$ coordinates.

Since we are considering elastic waves in a solid, the right member of the Lamé equation (3) should be replaced by the corresponding components of the inertia forces $F_{i n}$ in a chosen elementary volume. For instance, in the $x$ direction:

$$
F_{i n, x}=\rho \frac{\partial^{2} U}{\partial \tau^{2}}
$$

where $\rho$ is the material density.

The boundary conditions accepted in the above model assume: 1) the absence of normal and tangent stresses on the top and side plate surfaces, and 2) zero vertical displacements on the bottom surface and forced harmonic vibrations in the stimulation point. The internal crack surface is characterized by non-linear boundary conditions. At the stage of the crack compression, one assumes no tangent stresses, and at the stage of the crack strain, the condition of zero normal stresses is added. These conditions can be easily achieved by assuming that the corresponding members in equation (3) are equal to zero. As the initial condition, displacements are assumed to be zero at $\tau=0$. Then, a displacement by $x$ at each following time step can be calculated by solving equation (3), and the same for coordinates $y$ and $z$. The surface vertical displacement $W$ in a stimulation point is as follows:

$$
W=A[1-\cos (2 \pi f \tau)] / 2
$$

where $A$ and $f$ are the vibration amplitude and frequency, respectively.

The heating power in a crack perpendicular to the $x$ direction is calculated by:

$$
P=\frac{k_{f r} \sigma_{x} S_{f r}}{\tau *} \int_{0}^{\tau^{*}}\left|\frac{\partial U}{\partial \tau}\right| d \tau
$$

where $k_{f r}$ is the crack wall friction coefficient, $\sigma_{x}$ is the stress normal to the crack surface, and $S_{f r}$ is the crack surface.

For the second step, a $3 \mathrm{D}$ heat conduction equation is numerically solved to calculate the temperature distribution in a sample [5].

\section{Modelling ultrasonic thermal NDT}

This paper describes some results of the numerical modelling of ultrasonic IR thermography procedures with an emphasis placed on the inspection of sandwich plates.

The ThermoSon computer program developed at Tomsk Polytechnic University, Russia, was used to optimise heating parameters in the detection of subsurface defects in composite materials [6]. The program allows for the analysis of transient heat conduction and ultrasonic wave propagation phenomena in solids.

Sandwich panels with metal sheets and a soft core are considered in the paper. The analyzed model of composite (or composite model) has a thickness of $10 \mathrm{~mm}$ and lateral size $100 \mathrm{~mm}$. Three defects (lateral size $10 \mathrm{~mm}$ and thickness of $1 \mathrm{~mm}$ ) air-filled were placed at various depths (directly under the metal plate, $2 \mathrm{~mm}$ and $3 \mathrm{~mm}$ ) below the surface of the model of the composite specimens (Fig.6).

Thermal and strength properties of the composite were taken from literature [7] and information from manufacturers (Tab.1). The most common heat-insulating layer of this type of composite materials are used: mineral wool, polyurethane foam and foamed polystyrene. The insulating layer is coated on both sides with a thin $(0.5-1 \mathrm{~mm})$ metal sheet. 


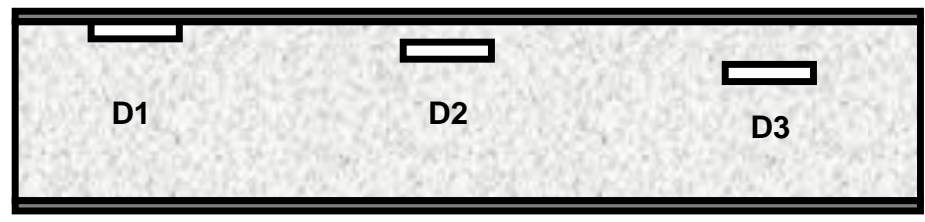

Fig. 6. Model of the composite specimens

Table 1. Thermal and strength properties of materials used in computer simulation

\begin{tabular}{|l|l|l|l|l|l|}
\hline Material & $\begin{array}{l}\text { Density } \\
\rho\left[\mathrm{kg} / \mathrm{m}^{3}\right]\end{array}$ & $\begin{array}{l}\text { Thermal } \\
\text { conductivity } \\
\lambda[\mathrm{W} / \mathrm{m} \cdot \mathrm{K}]\end{array}$ & $\begin{array}{l}\text { Specific heat } \\
\mathrm{C}_{\mathrm{p}}[\mathrm{J} / \mathrm{kg} \cdot \mathrm{K}]\end{array}$ & $\begin{array}{l}\text { Young's } \\
\text { modulus }[\mathrm{Pa}]\end{array}$ & Poisson ratio \\
\hline Polyurethane & 38 & $\leq 0.022$ & 1460 & $2.5 \cdot 10^{5}$ & 0.35 \\
\hline Mineral wool & 115 & $\leq 0.039$ & 750 & $4.2 \cdot 10^{\prime}$ & 0.20 \\
\hline Foamed polystyrene & 16 & $\leq 0.037$ & 1460 & $1.5 \cdot 10^{6}$ & 0.20 \\
\hline Steel & 7850 & 50 & 470 & $2.1 \cdot 10^{11}$ & 0.33 \\
\hline Air (thin gap) & 1.2 & 0.07 & 1005 & - & - \\
\hline
\end{tabular}

\section{Experimental testing}

The phenomenon of mechanical hysteresis seemed to vanish in the range of typically used ultrasonic frequencies and electrical powers (from 20 to $40 \mathrm{kHz}$ and up to a few $\mathrm{kW}$, respectively) [8, 9]. Therefore, a sound composite remained 'cold' during stimulation, while noticeable temperature signals appeared in defective areas due to internal friction. The experiments at MIAT were fulfilled by means of FLIR SC 7600 IR imager (image format $320 \times 256$, acquisition frequency $5 \mathrm{~Hz}$, up to 1600 images in a sequence). A continuous ultrasonic stimulation was performed with a piezoelectric unit at the frequency of $30 \mathrm{kHz}$ with the power from 60 to $100 \mathrm{~W}$ (maximum allowed power up to $2 \mathrm{~kW}$ ). Fig. 7 presents the setup used for thermographic tests with ultrasonic thermal stimulation. Due to hardware limitations in our research we could only use a frequency of $35 \mathrm{kHz}$.

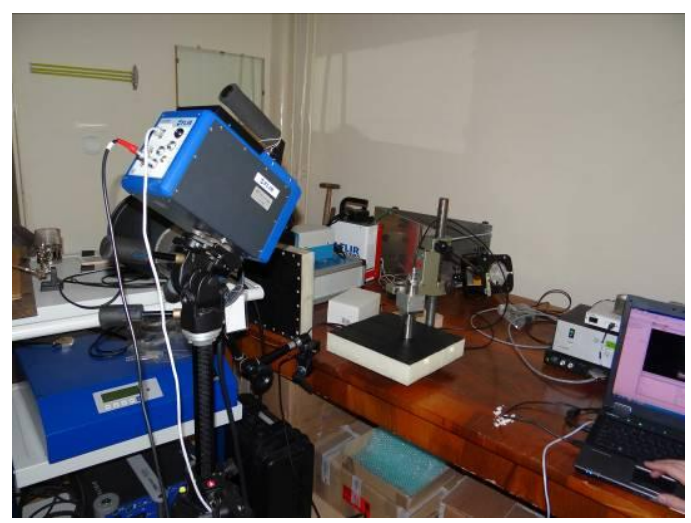

Fig. 7. Experimental setup and tested sample

Experimental testing included samples of sandwich plates containing defects in the type of delamination, cracks and blisters. Samples with three types of core material and thin, metal, light-profiled facings were analyzed. Sandwich panel with a polyurethane foam core was $60 \mathrm{~mm}$ thick and coated on both sides by steel sheets with a thickness of 0.6 $\mathrm{mm}$, filled with mineral wool had a thickness of $100 \mathrm{~mm}$ and was covered by steel sheet of thickness of $0.6 \mathrm{~mm}$, too. The last samples with a foamed polystyrene core had a thickness of $108 \mathrm{~mm}$. In this case a cover steel sheet had a thickness of $1 \mathrm{~mm}$. The steel sheet covering the core in tested samples is trapezoidal metal sheet with a low profile height of about $1 \mathrm{~mm}$.

\section{Results}

The Fig. 8 and 9 present selected results of computer simulations, the ability to detect defects that are directly (D1) under a steel sheet covering the core of sandwich panel. The results obtained with the generation of ultrasonic for 5 seconds. The simulation was performed for four different frequencies of ultrasound ( $35 \mathrm{kHz}, 30 \mathrm{kHz}, 25 \mathrm{kHz}$ and $20 \mathrm{kHz}$ ) 
most frequently used in this type of tests. Fig. 8 shows the results for the model of sandwich panel with a polyurethane foam core. As seen from illustration of the changes of temperature on surface of sheet above the defect increase of signal maximum temperature is from about $1.5^{\circ} \mathrm{C}$ for frequencies of $35 \mathrm{kHz}$ up to about $2.5^{\circ} \mathrm{C}$ for $20 \mathrm{kHz}$. It follows that a more suitable for detection of defects is the use of a lower frequency value.

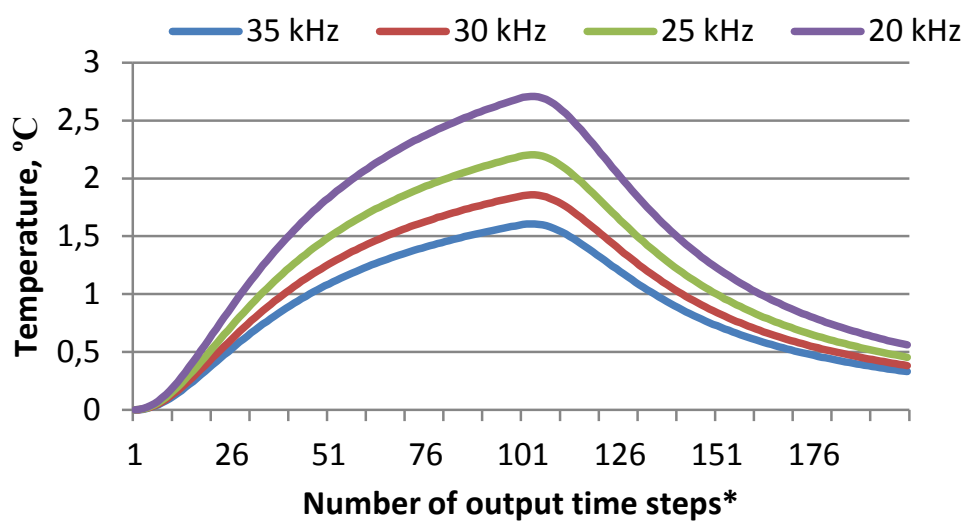

Fig. 8. Change in the signal temperature above the defect D1 at different frequencies for a model of sandwich plate with a polyurethane foam core ('time step $0.05 \mathrm{~s}$ ).

Fig. 9 shows results obtained for sandwich panel with a mineral wool core. In this case, the temperature increase above the defect at the same simulated, is significantly lower and ranges from about $0.14^{\circ} \mathrm{C}$ for $35 \mathrm{kHz}$ to $0.16^{\circ} \mathrm{C}$ for $20 \mathrm{kHz}$.

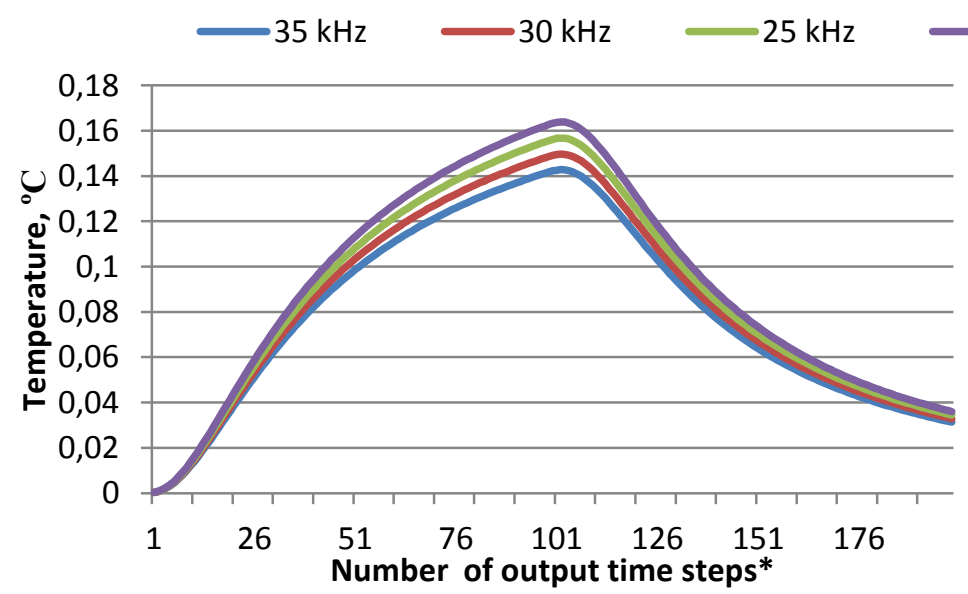

Fig. 9. Change in the signal temperature above the defect $D 1$ at different frequencies for a model of sandwich plate with a mineral wool core ( ${ }^{*}$ time step $0.05 \mathrm{~s}$ ).

The calculations for defects deeper locations (D2 and D3) at the surface of composite revealed that the increase in temperature on the surface of sheet above defect is too small to be detected.

Some examples of defects detected during experimental tests were presented in Fig. 10 and 12. Fig. 10 shows thermogram of the test on sample with a polyurethane foam core. The detected failure has the form of lack of the core material directly under the cover sheet. This thermogram shows only a part of the defect, because the defect is located under the flat parts of profiled edge of the cover separated. The edges limit the propagation of ultrasonic waves. The waves may propagate mainly flat element of profiled sheet limited on both sides edges. To detect all defects, ultrasound in following components of flat covering the core have to be generated. If the defect is located at adjacent to each other parts of its flat plate then it be detected by ultrasound generation in both adjacent components.

Fig. 11 shows a temperature distribution on the surface of a sheet above the defect detected on thermogram Fig. 10. Registered maximum temperature rise of about $1.5^{\circ} \mathrm{C}$. This value is comparable to the result obtained during the computer simulation shown in Fig. 8 (temperature changes at the frequency of $35 \mathrm{kHz}$ ). 


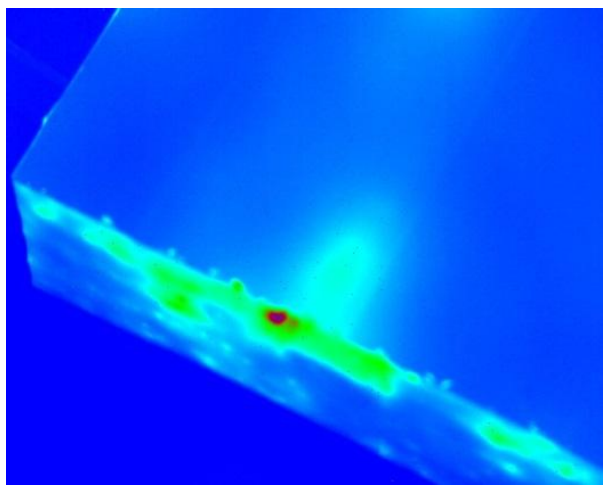

Fig. 10. Experimental result - thermogram

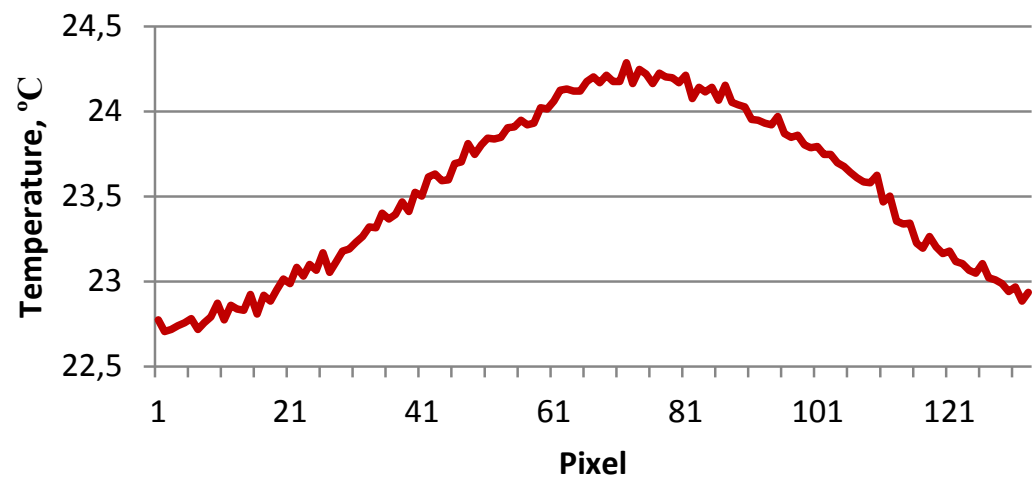

Fig. 11. Temperature changes on the surface of the sheet above the defect visible on the thermogram Fig. 10

When defects cause too large temperature rise, which is easily distinguishable on thermograms, then you should use a special image (thermogram) processing. This case is shown in Fig. 12.

A source sequence was processed by applying some algorithms known in pulsed thermal nondestructive testing, i.e.: normalization (N), polynomial fitting (PF), pulse phase thermography (PPT), Fourier analysis (FA), principal component analysis (PCA), and correlation analysis (CA). The efficiency of data processing techniques is discussed. Figure 12 shows the source image (a better thermogram) of detected defects in a sandwich panel with extruded polystyrene foam core and steel sheets after ultrasonic IR thermographic testing (Fig. 12a), and the image after analysis of the PCA (Fig. 12b).

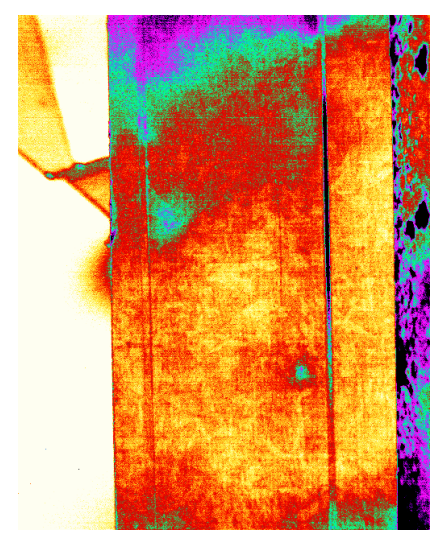

a)

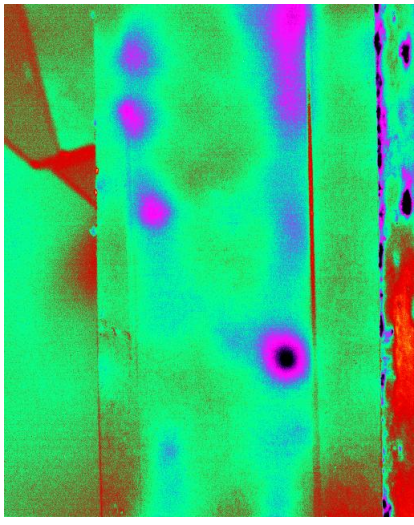

b)

Fig. 12. Experimental results 


\subsection{1/qirt.2016.132}

\section{Conclusions}

The numerical simulations that were carried out confirmed by experimental research showed that the composites used in thermal insulation systems of buildings like sandwich panels covered with trapezoidal metal sheets are difficult objects for research with thermography methods. The coverage, which thermal parameters are significantly different from the parameters of the core materials has decisive influence on effectiveness of detection.

Use of the ultrasonic thermographic method allows detection of defects as delamination, cracks and blisters located directly under the sheet covering the composite. It is very difficult to detect cracks of the core running from composite coating into the material. In many cases, special image analysis allows detection of defects that are difficult to locate on the source thermograms. Results that obtained by heating with ultrasound are significantly better than the optical heating source (e.g. heating and flash lamps, lasers etc.).

Furthermore, in our work we focused on studies using other frequency ultrasound thermal stimulation than used in experimental tests presented in this paper. As shown by numerical calculations it should improve the detection of defects.

\section{REFERENCES}

[1] Katunin A., Chuda-Kowalska M., "Assessment of Internal Damages in Sandwich Panels via Active Thermography". Engng. Trans. Vol. 63, No 2 pp. 171-180, 2015.

[2] A.A. Samarsky A. A., Nauka Publ., Moscow, 1989, p. 619 (in Russian).

[3] Homma C., Rothenfusser M., Baumann J. et al., "Review of Quantitative Nondestructive Evaluation" Vol. 25, American Institute of Physics, CP820, 2006, pp. 566-573.

[4] Lourier A. I., Nauka Publ., Moscow, 1970, p. 940 (in Russian).

[5] Vavilov V., Swiderski W., Derusova D., "Ultrasonic and optical stimulation in IR thermographic NDT of impact damage in carbon composites", Quantitative InfraRed Thermography Journal, Vol.12, No 2, 2015, pp.162-172

[6] ThermoSon, Operational Manuel, Innovation Ltd., Russia, Tomsk, 2015

[7] Maldague X. P., "Theory and Practice of Infrared Technology for Nondestructive Testing" Wiley-Interscience Publication, 2001

[8] Busse G., Gleiter A. and Spiessberger D., "NDE Using Lockin-Thermography: Principle and Recent Developments", Proceeding of NDTMS-2011, Springer, RILEM Bookseries, Istanbul, pp 627-631, 2011

[9] Swiderski W., Vavilov V., "Ultrasonic IR thermographic inspection of graphite epoxy composite: a comparative study of piezoelectric and magnetostrictive stimulation", Opto-Electronics Review, Vol. 23 (1), 2015, pp. 33-36 